\title{
BMJ Open Using patient feedback to drive quality improvement in hospitals: a qualitative study
}

\author{
Simone Berger (D) , Ana Maria Saut (D), Fernando Tobal Berssaneti (C)
}

To cite: Berger S, Saut AM, Berssaneti FT. Using patient feedback to drive quality improvement in hospitals: a qualitative study. BMJ Open 2020;10:e037641. doi:10.1136/ bmjopen-2020-037641

- Prepublication history for this paper is available online. To view these files, please visit the journal online (http://dx.doi. org/10.1136/bmjopen-2020037641).

Received 12 February 2020 Revised 06 July 2020 Accepted 25 September 2020

D Check for updates

(C) Author(s) (or their employer(s)) 2020. Re-use permitted under CC BY-NC. No commercial re-use. See rights and permissions. Published by BMJ.

Department of Production Engineering, University of São Paulo, Sao Paulo, Brazil

Correspondence to Mrs Simone Berger; siberger@uol.com.br

\section{ABSTRACT}

Objectives Although different forms of patient feedback are available, their use in hospital management is still limited. The objective of this study is to explore how patient feedback is currently used in hospitals to improve quality.

Design This is a qualitative exploratory multiple case study. Data collection included nine interviews, of an average duration of $50 \mathrm{~min}$, conducted between March and June 2019. Additionally, a document and secondary data analysis were performed.

Setting This study was conducted in three Brazilian hospitals selected for their solid patient feedback practises.

Participants Managers from the customer service, quality, nursing, operations, projects and patient experience departments of the three hospitals.

Results Despite literature suggesting that organisational objectives regarding patient feedback are not clear, data show that there is managerial concern regarding the promotion of an environment capable of changing according to patient feedback. In these instances, organisational processes were structured to focus on patients' feedback and its receipt by the staff, including a non-punitive culture. Several patient feedback forms are available: voluntary events, patient surveys and informal feedback. Instruments to measure patient feedback focused on specific aspects of healthcare, to identify and clarify the problems for addressal by the management. The net promoter score was the main strategic indicator of patient feedback, used to assess the impact of improvement action.

Conclusions The hospitals had established objectives that valued the patient's perspective. Involvement of the health team, availability of different channels for feedback and the use of quality tools are considered a good basis for using patient feedback to drive quality improvement.

\section{INTRODUCTION}

Patient experience is considered as one of the pillars of quality in healthcare, along with patient safety and clinical outcomes. ${ }^{1} 2$ Providing feedback on the experience of patients and encouraging the healthcare team to address the identified problems, thus improve quality, is the best way to ensure that services meet objectives. ${ }^{3}$ However, despite the availability of different

\section{Strengths and limitations of this study}

Hospitals were selected for their solid patient feedback practises.

- Data collection consisted of interviews with managers from areas related to patient feedback.

- The definition of the research protocol was based on the previous literature, thereby consolidating the categories studied.

- In addition to the interviews, we conducted a document and secondary data analysis.

- The main limitation of this study is that it is based on just nine interviews in three accredited large hospitals, thus, it only begins to answer the research question.

forms of patient feedback, their use in hospital management is still limited. ${ }^{4}$

Patient feedback can be defined as the recording of the patient's perspective on the quality of care received for the purpose of learning how to improve processes and the patient experience. ${ }^{5}$ The term 'patient feedback' can be used to describe several types of formal feedback, including levels of patient satisfaction, experience, views and assessments of care in terms of accessibility, continuity and quality. ${ }^{6}$

Donabedian $^{7}$ described the three roles that the patient could play in healthcare quality in terms of: 'contributors, targets and reformers'. In the 'contributors' role, patients define quality, evaluate quality and provide information that permits others to assess it. Consumers as the 'targets' of quality are defined by their engagement in the production and control of care, and the 'reformer' role is related to direct participation through administrative support and political action.

Establishing a formal communication process with patients may be considered the first stage of patient involvement because obtaining feedback can provide the information needed to promote improvements in internal processes and support strategic 
planning. ${ }^{8}$ According to Hernan et al, ${ }^{9}$ implementing a patient feedback system involves the collection of feedback from patients through research and the analysis of the results by a designated team that later reports back to the health team for inclusion in the action plan.

Few studies have addressed how and when patients should be involved, or how to provide a friendly environment for their involvement, with the aim of improving health outcomes and the care experience. ${ }^{10}$ Managers need this information so that they can review their practises and ensure that these are focused on actions that lead to quality care. ${ }^{4}$

Although the misuse of patient feedback surveys has been widely reported in the literature, there are many related challenges that still need to be assessed to demonstrate the complexity of the topic and enable practical solutions to make the surveys useful. ${ }^{11}$ From this perspective, Berwick ${ }^{12}$ states that quality science offers valuable alternatives to the hostility and confusion that inspections, awards and punishments can cause. The use of quality tools in health services can provide substantial improvements, and the science of improvement should integrate a training curriculum for healthcare staff and managers.

This underlying study was motivated by the potential use of patient feedback in guiding quality improvement actions. The central question of this study is 'How do hospitals use patient feedback to drive quality improvement?'

\section{METHODS}

This research uses an exploratory, qualitative and multiple case study approach. Case study research promotes our understanding of real-world phenomena and assumes that this understanding encompasses pertinent contextual conditions. ${ }^{13}$

We studied three accredited hospitals located in São Paulo, Brazil, which were most likely to help answer the research question and provide solid patient feedback practises. The accreditation programmes support hospitals to conduct quality management processes. ${ }^{14}$ São Paulo is an important economic hub in Latin America and is also a reference in health services.

Data collection included three interviews in each hospital, with an average duration of $50 \mathrm{~min}$, conducted between March and June 2019. The criteria for inclusion were that the participant was qualified to represent the hospital to clarify issues related to patient feedback and had at least 6 months of experience in the hospital. Managers were interviewed in areas related to patient feedback such as the customer service, quality, nursing, operations, projects and patient experience.

Data was consistent since most of the practises reported by the hospitals were coincident, with only a few particularities described. These results reinforce and enrich the categories found in the literature review: (1) objectives, (2) enabling environment, (3) forms of patient feedback, (4) biases, validity and reliability, (5) quality improvement methodologies, and (6) organisational processes involved.

We defined these categories based on the literature. Lee $e t a t^{4}$ conducted a case study in which they analysed objectives, context, forms, organisational processes and use. Baldie $e t a l^{6}$ conducted a structured literature review and defined context, mechanisms and results. Hernan et at ${ }^{\theta}$ explored data collection, learning, feedback provided to the health team, action plans and implementation using a qualitative-quantitative approach. Snyder and Engström ${ }^{10}$ addressed forms of involvement, training of the health team, patient education, organisational factors and consequences of involvement in their narrative literature review.

The interviews were composed of open-ended questions in the identified categories and were recorded. The questionnaire was tested in a pilot case to estimate the required execution time and to validate the protocol (table 1).

The interviews were transcribed immediately after they were conducted, and the transcripts were imported into
Table 1 Research protocol

\begin{tabular}{|c|c|}
\hline Theme & Questions \\
\hline \multirow[t]{4}{*}{ 1: How does the hospital get feedback from patients? } & 1.1 What are the forms of patient feedback? \\
\hline & $\begin{array}{l}1.2 \text { How does the hospital maintain an enabling environment so } \\
\text { that the healthcare staff receive feedback from patients? (explore } \\
\text { organisational goals and predisposition) }\end{array}$ \\
\hline & $\begin{array}{l}1.3 \text { How does the hospital maintain an enabling environment so that } \\
\text { patients provide feedback? }\end{array}$ \\
\hline & 1.4 How does the hospital assess validity and reliability? \\
\hline \multirow{2}{*}{$\begin{array}{l}\text { 2: How are quality improvement actions based on } \\
\text { patient feedback implemented? }\end{array}$} & 2.1 How are quality improvement methodologies used? \\
\hline & $\begin{array}{l}\text { 2.2 What improvements have been implemented based on patient } \\
\text { feedback? }\end{array}$ \\
\hline
\end{tabular}

Source: The authors. 
Table 2 Characteristics of the analysed hospitals

\begin{tabular}{llll}
\hline Case & A & B & C \\
\hline Type & General hospital & General hospital & General hospital \\
Size & Large & Large & Very large \\
Number of Interviews & 3 & 3 & 3 \\
Areas covered & $\begin{array}{l}\text { Customer service, quality } \\
\text { and nursing }\end{array}$ & $\begin{array}{l}\text { Operations, customer service and } \\
\text { superintendence }\end{array}$ & $\begin{array}{l}\text { Customer service, projects and } \\
\text { patient experience }\end{array}$ \\
\hline
\end{tabular}

Source: The authors.

NVivo 11 software, where the predefined categories had been recorded. The excerpts from the interviews were then correlated to the categories allowing data consolidation by category. The results enrich the literature by highlighting possible challenges and opportunities.

In addition to the interviews, we conducted a document analysis that included reviewing patient feedback forms, data consolidation reports, action plans, process standards and protocols for implementing improvements, as well as hospital secondary data, such as the institutional websites, social networks and the service site for registering consumer complaints.

\section{Patient and public involvement}

No patients were involved.

\section{RESULTS}

Our study captures good practises for patient feedback observed in three accredited, general hospitals in São Paulo, Brazil; two were large sized (150-500 beds) and one was very large with more than 500 beds, as described in table 2.

The respondents were managers at the hospital, with postgraduate education, who were working there for a minimum of twoyears (table 3 ).

After each interview, the respondents were asked to show documentary evidence. The web pages of the hospitals aimed at registering patient feedback reported positive and negative comments and suggestions, which were analysed. On the internet, patients also exchange messages with the hospitals through social networks such as Facebook and Twitter.

Data show that the objectives for obtaining patient feedback are clear, and there is managerial concern regarding the promotion of an environment amenable to changing according to patient feedback. It was seen that several patient feedback forms are available and that organisational processes are focused on patients' feedback and its receipt by the staff. Mechanisms to analyse the validity, reliability and biases are considered to define and analyse the forms of feedback, and quality improvement methodologies are used to define action plans, projects and indicators.

Respondents mentioned examples of initiatives taken by the hospitals to improve patient health, based on patient feedback. For example, projects were defined according to quantitative analysis, such as meal temperature and variety, waiting time, parking fees, administrative processes on reimbursement and hospital discharge. Conversely, a few complaints by patients from the qualitative analysis also led to direct action by the hospitals with impact to several patients, such as offering blankets to patients and controlling luminous intensity control in the examination rooms.

Empirical evidence was found for all categories studied. Table 4 presents statements to exemplify each one, and in sequence, each category is detailed.

\section{Category 1: objectives}

It was clear that the hospitals had established objectives that valued the patient's perspective. However, despite efforts to create a non-punitive culture, it was difficult to raise awareness among managers, and the health team was particularly resistant.

'It is about implementing a culture. A culture is not a design, it is not a flow, nor a system. A culture is

\begin{tabular}{|c|c|}
\hline Characteristic & Observation \\
\hline Education & $\begin{array}{l}\text { Specialisation course and/or master's } \\
\text { degree }\end{array}$ \\
\hline \multirow[t]{2}{*}{ Profession } & $\begin{array}{l}\text { Customer service area: administrator (1), } \\
\text { physiotherapist (1), hospital administrator } \\
\text { (1) }\end{array}$ \\
\hline & $\begin{array}{l}\text { Quality, nursing, operations, projects, } \\
\text { patient experience areas: nurses } \\
\text { (2), nutritionists (2), physician (1), } \\
\text { administrator (1) }\end{array}$ \\
\hline Position & Director (1), managers (4), supervisors (4) \\
\hline \multirow[t]{4}{*}{ Time in job } & 1 respondent over 30 years in that function \\
\hline & $\begin{array}{l}2 \text { respondents between } 15 \text { and } 30 \text { years in } \\
\text { that function }\end{array}$ \\
\hline & $\begin{array}{l}4 \text { respondents between } 5 \text { and } 15 \text { years in } \\
\text { that function }\end{array}$ \\
\hline & $\begin{array}{l}2 \text { respondents between } 2 \text { and } 5 \text { years in } \\
\text { that function }\end{array}$ \\
\hline
\end{tabular}

Source: The authors. 
Table 4 Categories of feedback in the cases under study

\begin{tabular}{ll}
\hline Category & Respondent \\
\hline $\begin{array}{l}\text { Objectives: objectives for obtaining patient } \\
\text { feedback are clear }\end{array}$ & Nursing manager of Case A \\
& $\begin{array}{l}\text { Customer service supervisor of } \\
\text { Case B }\end{array}$
\end{tabular}

Statements from the interview

'One of the pillars of the company is customer satisfaction; that is what we always have to look out for'.

Case B

'Feedback aims exactly to explore what is hidden and to know how to access it. When a patient complains, you must try to understand them, because this is an opportunity, it is a gold mine. We train our teams, for this you must have the know-how, skills, seniority'.

Projects manager of Case C 'The patient's experience is detailed on our strategic map, so that the entire institution knows that the patient's experience will contribute to our sustainability'.

Enabling the environment: Staff perspective institution provides an environment to enable

change according patient feedback Case A

Customer service supervisor of Case B

Projects manager of Case C

Forms of patient feedback: several forms of patient feedback available

Customer service supervisor of Case A

Customer service supervisor of Case B

Customer service supervisor of Case C

Biases, validity, and reliability: aspects considered Nursing manager of Case A to define and analyse patient feedback

Operations manager of Case B

Patient experience superviso of Case $\mathrm{C}$

Quality improvement methodologies: methodologies and tools are used to drive quality improvement

Quality manager of Case A

Customer service supervisor of Case B

Patient experience superviso of Case C

Organisational processes: processes are defined to support quality improvement through patient feedback
Quality manager of Case A

Director of Case B

Patient experience supervisor of Case C
'We are able to mediate the situation, so the customer service is not punitive. We do not say, 'Oh you were wrong, we will punish you with a warning, or with a suspension, we will reduce your hours in the clinic'. Nothing like that; our customer service is educational, so we show the managers what needs to be improved'.

'The staff understand that it is really through the customer that we can understand and then implement what they are expecting'.

'Even if an individual member does not like it, the staff know that patient feedback is important and that it will have to be that way. We will have to understand more of what our patients think of us, to be super efficient in identifying and correcting what they are asking for'.

'There are posters on every floor encouraging patients to contact us, whether by telephone, email or requesting us to come to their bedside or the emergency room. Therefore, we encourage the patient to contact us as much as possible'.

'Patients are involved in their own care, so they know. People are very enlightened with Doctor Google, they know everything. Today, people who do not express themselves are very rare. They sometimes complain, 'I thought they did something wrong to me".

'We display the ombudsman's phone number, and advise, 'If you need help anytime, we are here, get in touch, you can write or call'. During hospitalisation, patients, or their family, call the ombudsman to include a neutral element, but most patients directly contact the health team'.

'Nowadays we have several communication channels available to the patient. We have a personal attendance service, telephone and email facilities. There is an online satisfaction survey, in which the patient can report his manifestation, complaint, appreciation or suggestions, as well as social networks'.

"The customer manifests in several ways. There is a form "Your Opinion" displayed in the hospital areas, personal attendant services, and email or social media links to Facebook and Twitter are available. Recently the hospital set up Instagram and Linkedln accounts as well. We have many sources of information. Even healthcare operators are a good source of information'.

'The reason for having several channels is that we are more accessible to the patient. However, it is also important to also have a department that is responsible for coordination, to record and forward the feedback, and action the interface between the areas'.

'Research adherence is high; I think half of the people respond. Those who respond, mostly have complaints. Those who do not want to complain, do not respond'.

'In healthcare, there is a bias in manifestations because people are fragile. This human relationship requires staff to have attributes such as agility, promptness, and the ability to listen'.

'We run a statistical analysis on the surveys. We have a whole sample calculation to analyse whether a problem is representative of that population. We do correlation and trend curve analysis. We work a lot on this research data'.

'If a problem occurs in many areas, we can use more consistent risk assessment tools, such as FMEA (Failure Mode and Effect Analysis), which is a failure model'.

'We use quality assessment tools like Ishikawa and Pareto diagrams, Plan, Do, Check and Action cycles; these tools are used regularly by the staff. Everyone knows it, that is how we make our action plans'.

'If the complaints relate to opportunities for improvement in the processes, the Operational Excellence Office will apply Lean methodology and analyse it'.

'The net promoter score may or may not be linked to variable remuneration, depending on the department. This is totally debatable, linking a goal of the department to indicators related to the quality of the care process. This is not good, because it can reduce the number of notifications'.

'All the comments come to us, through the ombudsman channel, whether it is a medical, management, administrative, or team conduct matter. We have weekly meetings with the management, to obtain a professional view on the patient's perspective, or on something that the patient said, but did not formally report'.

'Everyone is aware of the problems, and the management says, 'What do you need us to support you with? For us to solve?' It is not just speech, or saying 'solve it yourself'. If there is a problem, the management says, 'Let us solve it together, what do you need our support for? Let us work together". 
characterised by a mass of consciousness about that subject within the institution. This is a challenge for those who work with improvement systems'. (Quality manager of Case A).

Factors such adequacy of accreditation and external benchmarking were viewed as structured ways that could improve the process of using patient feedback in these institutions.

'This work of certification was very important for people to understand that we are here for the client, not only for the process, but for the client to whom you are providing this process. This brings out a whole range of possibilities. I think that the accreditation helped to contribute that'. (Operations manager of Case B).

\section{Category 2: enabling the environment for patient feedback}

From an organisational perspective, the theme of patient feedback appeared to take on strategic importance. The data indicate a focus on promoting a non-punitive culture along with a commitment to promote action from this perspective. The training and educational campaigns provided to the hospital teams aim to promote a favourable environment. 'We cannot improve the customer service or patient experience areas if we do not have much support from the senior management'. (Customer service supervisor of Case C).

From the perspective of the healthcare team, we found that the involvement of the health team was considered a fundamental pillar, without which the implementation of quality improvements would be compromised, restricting patient feedback to timely and unstructured corrective actions. 'The hospital works hard for the people; the greatest value for the institution is our health team, to serve the patients and staff well'. (Director of Case B)

The problems to enabling the environment for patient feedback were overconfidence, high team turnover and the importance of managing positive comments. Respondents stated that compliments are not treated with the same relevance as complaints. Empirical evidence also shows that instituting compliment-based awards may generate inappropriate conduct from the team if they specifically request the patients to provide the compliment.

Conversely, the factors that enabled the environment were: keeping the staff informed about the use of feedback from patients, ensuring their awareness of the process, along with support from the institution for staff well-being. 'The hospital subsidises several training courses for the team, according to their area of work, so our staff has the possibility to grow culturally and professionally'. (Operations manager of Case B).

From the patient's perspective, the respondents indicated that the greatest difficulties in providing feedback were lack of knowledge about the care process and the fear of being interviewed or of expressing opinions during care delivery. 'The patient is well informed by staff about the feedback processes; for example, in providing feedback, the patient is participating in the process of careprovision, the patient does their part and the hospital reciprocates'. (Customer Service supervisor of Case B).

\section{Category 3: forms of patient feedback}

The forms of feedback were divided into three types that could be conducted during or after care delivery.

- Voluntary events include voluntary registration through channels available at the customer service of the hospital, such as face-to-face feedback, telephone, email, letter, internet form and registration on social networks such as Facebook and Twitter.

- Patient surveys: The institution approaches patients for their perspectives on their care through annual online sample surveys, telephone surveys or online surveys sent to patients within 24 hours of care delivery, specifically for each health service or disease.

- Informal feedback: Patients express their opinions to the care team verbally.

'If nurses go into the room and solve the problem, it is okay. However, if this occurrence was not reported, we could not study it, and afterwards, we also could not study the recurrence. That is the only way you can handle action plans'. (Customer Service supervisor of Case C).

The results highlight the importance of several channels being made available during the whole patient journey, in order to stimulate the patient manifestation. In contrast, there is staff concern about implementing new technological channels and overloading the feedback processes thereby, damaging credibility.

\section{Category 4: biases, validity, and reliability of surveys and feedback}

Data show that factors related to patients' manifestations, biases, validity and reliability of surveys are considered, especially regarding the patient's health and emotional condition. This is the reason why the instruments to measure patients' feedback were focused on specific aspects of healthcare, to clarify the real management problem to be addressed. The hospitals analysed have technological solutions to focus feedback on specific healthcare points, according to the patient.

'When dissatisfaction is very high, people are reactive. They are trying to convey something else. They are angry because someone is dying or because their child has a disease that may not be cured. Then, I guess we also must understand that'. (Quality manager of Case A).

\section{Category 5: quality improvement methodology}

With the support of quality improvement methodologies, the patient's perspective (obtained by several means) is recorded and stratified. In general, the cases are grouped 
into one-off problems, recurrent chronic problems and opportunities for improvement, which guide the creation of new projects or relate to existing ones.

The net promoter score (NPS) was used as a strategic indicator in these hospitals. It consists of a loyalty indicator developed by Bain and Company to analyse the impact of improvement actions on organisations. Using the question 'Would you recommend this institution to a relative or friend?', it is possible to calculate the factor by subtracting the percentage of promoters by the percentage of detractors. ${ }^{15}$

'We use science and the data to our advantage. If you want to work with the NPS, this is how you do it. The board understands that this is a strategic indicator, but other indicators should be considered alongside. NPS does not work by magic'. (Patient experience supervisor of Case $\mathrm{C}$ ).

All institutions reported using the Plan, Do, Check, and Action cycle, quality tools such as the Ishikawa and Pareto diagrams, and risk management tools. Respondents also mentioned that projects should be prioritised by the subject. Issues related to patients' preferences, such as food and hospitality, are addressed, but actions that can prevent harm to the patient should be treated as a priority.

To foster a favourable environment, the hospitals promoted a non-punitive culture of safety, aimed at receiving and addressing interactions in a structured manner so that they could guide preventive action. Our study highlights that there are institutional areas of quality focussing on patient safety, using patient and family interactions as barriers to adverse events. We also discuss that processes must be established and made clear to the health team so that involuntary errors and violations could be distinguished.

\section{Category 6: organisational processes}

Organisational processes should be defined to support quality improvement actions through feedback from patients, senior management, managers and the care team. If not, there is a loss of credibility, jeopardising the maintenance of a friendly environment and compromising the process of patient feedback, in addition to causing financial losses due to the misuse of resources allocated to the systems for the application of surveys, the user engagement team and the analysis of manifestations.

'Customer service participates in our leadership meetings, where all the managers are present. The supervisor reports a general summary of the actions and the improvements that have been made'. (Operations manager of Case B).

Patient feedback indicators were considered independent dimensions of quality. They were defined and periodically monitored by senior management and were included in the agenda of managers at various hierarchical levels. The NPS was mentioned as the main strategic indicator of patient feedback to assess the impact of improvement actions.

'The strategic indicator of the institution is the NPS, which I think everyone already uses. However, senior management and the entire institution understand that it is not only a channel that allows us to see the perception of the patient'. (Patient experience supervisor of Case $\mathrm{C}$ ).

The percentage of patients complaining in relation to the total number of treated patients was also used as an indicator. However, it was also mentioned that the few complaining patients could damage the image of the hospital to the extent that they could communicate their dissatisfaction to others. Our study reveals the importance of establishing suitable indicators to direct appropriate actions based on patient feedback.

Management indicators based on research on patients' experience, emphasizing priority areas of action, were used to define improvement actions. The manifestations registered by the customer service were broken down by area, responsible party and motive, and then related to existing projects or the generation of new ones. The effectiveness of the projects was analysed by monitoring the evolution of the research indicators and the recurrence of the manifestations. Finally, qualitative patient reports generated ideas and promoted improvement actions, where a few manifestations could impact several patients.

\section{DISCUSSION}

This study explored how hospitals use patient feedback to guide quality improvement, consolidating good practises through the categories observed in three accredited hospitals. The categories were defined as: (1) objectives; (2) enabling environment; (3) forms of patient feedback; (4) biases, validity and reliability; (5) quality improvement methodologies and (6) organisational processes involved. The data show that patient feedback is addressed strategically in these hospitals, and the processes are widespread at the managerial and care levels, as reported by several studies. $^{416-21}$

The literature suggests that the objectives for using patient feedback for quality improvement may not be clear. ${ }^{16} 1722{ }^{23}$ However, in contrast, we found evidence that the strategic objectives are in fact clear, and that the top management considers them to be important monitoring indicators that promote actions aimed at improving quality. However, regarding the difficulties in raising awareness among managers and healthcare teams, this study was in agreement with the literature. ${ }^{22} 24$

Our findings corroborate the literature relating staff involvement to patient feedback. Involving staff is the most challenging aspect in the promotion of a favourable environment. ${ }^{22}$ People are more likely to act if they believe. This aspect can stimulate behavioural change if the team considers the process, the instrument, and the conduct of the research to be reliable. ${ }^{2425}$ 
According to Nordin et $a l^{26}$, quality of service improvement is based largely on negative comments, while positive comments are usually neglected. We found evidence on this, and on the complexity in establishing an indicator related to compliments and complaints. Goals related to the reduction of complaints may inhibit feedback registration, jeopardising the achievement of the objectives of the feedback process.

The factors related to enabling the environment were divided into categories found in the literature: acceptance and belief in feedback from patients by the health team, autonomy for decision-making and the well-being of the health team. ${ }^{1722242728}$ Besides the barriers found in the literature relating to low credibility of patients' feedback by the team, our study highlights other difficulties of staff overload, healthcare team overconfidence, and high turnover in the team.

From the patient's perspective, our results agreed with the literature. ${ }^{1729} 30$ Patients are more likely to express their opinion if they are aware of the care process and if they find the feedback process confidential, reliable and transparent.

The forms of patient feedback observed in our study are aligned with those observed in the literature. ${ }^{1731-35}$ Emphasis on addressing feedback through social networks and the registration of informal feedback, was also observed as reported in the literature ${ }^{36-38}$ so that more patients could express themselves through applications such as WhatsApp.

Our study also emphasises the importance of informal feedback, which is poorly reported in the literature. ${ }^{39}$ Evidence shows that patient feedback to the healthcare team during care is rarely studied, despite having the potential for rapid improvement action. However, difficulties during registration were attributed to the work overload of the care team.

The validity and reliability issues reported in the literature $^{2240}$ were confirmed by the hospitals. The biases in the manifestations were also analysed. In the study, patient feedback was grouped into patient expectations, ${ }^{51}$ nonresponse, ${ }^{42}$ health status, ${ }^{42}{ }^{43}$ appropriate time and the focus on an event or specific interaction in the surveys with patients. ${ }^{41} 4344$ The results enrich the literature regarding the necessity of a qualitative analysis on the patient's health condition because the real cause of dissatisfaction could be hidden. Besides, a single unfavourable event can cause several other complaints that should be considered in patient feedback analysis.

This study identifies four opportunities for improving healthcare management practises. First, in addition to promoting awareness of the importance of patient feedback and team well-being, as reported in the case study, one suggestion is to promote training on human errors ${ }^{456}$ and mental biases, ${ }^{47}$ with the possibility of taking on a more scientific approach.

Second, the indicators should be defined using quality improvement methodologies and scientific criteria, ${ }^{48}$ providing clarity on the processes, expected results, metrics to define goals, and prioritisation of the main actions on the patient experience.

Third, the use of technologies, such as applications for recording manifestations, may be an alternative to broaden the perspective of patients at the time of care. However, institutions should implement them gradually, as recommended by some studies, ${ }^{49}$ and structure the areas for rapid mediation and treatment of results, supported by quality improvement methodologies. Further, another suggestion is to consider the mechanisms needed to record informal feedback by the health team at the time of care. New forms of patient feedback can be implemented through an analysis of the factors indicated by Baldie et $a t^{6}$ considering cost, feasibility, acceptance and educational impact, in addition to data validity and reliability.

\section{Limitations}

The limitations of this study relate to its limited scope, as it only begins to answer the research question. This study does not have the scope to provide complete evidence, but only to increase the body of knowledge and initiate discussions on the subject, which future studies can then explore.

The methodology and the selection criteria of the hospitals and the respondents are also limitations, that may have influenced the collected data. Strong quality improvement practises through patient feedback were observed in the accredited hospitals, but different processes could also be observed in other similar units of analysis or other contexts, such as small and mediumsized hospitals, specialised and public hospitals, and several initiatives aimed at improving patient experience through feedback.

According to Yin, ${ }^{13}$ exploratory studies should identify issues for further research. Thus, more comprehensive research on hospitals with different characteristics is needed as well as quantitative studies on the indicators of quality improvement through patient feedback.

Acknowledgements The authors thank the hospitals that participated in this research, without which this work would not have been possible. We also thank the University of São Paulo, Coordination for the Improvement of Higher Education Personnel and the National Council for Scientific and Technological Development.

Contributors SB was responsible for collecting data and draughting the manuscript. AMS revised and edited the manuscript. SB, AMS and FTB contributed to the analysis of the data. All authors have read and approved the final manuscript.

Funding The authors have not declared a specific grant for this research from any funding agency in the public, commercial or not-for-profit sectors.

Competing interests None declared.

Patient and public involvement Patients and/or the public were not involved in the design, or conduct, or reporting, or dissemination plans of this research.

Patient consent for publication Not required.

Ethics approval Prior to data collection, our project was approved by the Research Ethics Committee of the School of Arts, Sciences, and Humanities of the University of São Paulo. All participants were informed of the objective, justification and purpose of the study, and signed an informed consent form.

Provenance and peer review Not commissioned; externally peer reviewed. 
Data availability statement All data relevant to the study are included in the article or uploaded as supplementary information. No additional data are available.

Open access This is an open access article distributed in accordance with the Creative Commons Attribution Non Commercial (CC BY-NC 4.0) license, which permits others to distribute, remix, adapt, build upon this work non-commercially, and license their derivative works on different terms, provided the original work is properly cited, appropriate credit is given, any changes made indicated, and the use is non-commercial. See: http://creativecommons.org/licenses/by-nc/4.0/.

\section{ORCID iDs}

Simone Berger http://orcid.org/0000-0003-0131-3749

Ana Maria Saut http://orcid.org/0000-0002-8775-7385

Fernando Tobal Berssaneti http://orcid.org/0000-0002-8604-1887

\section{REFERENCES}

1 Committee on quality of health care in America, and Institute of medicine staff. Crossing the quality chasm: a new health system for the 21st century. Washington, DC: National Academies Press, 2001.

2 Berwick DM. What 'patient-centered' should mean: confessions of an extremist. Health Aff 2009;28:w555-65.

3 Coulter A. Patient feedback for quality improvement in general practice: a mixed bag of poorly evaluated methods leaves patients frustrated, and doctors little wiser. BMJ 2016;352:i913.

4 Lee R, Baeza JI, Fulop NJ. The use of patient feedback by hospital boards of directors: a qualitative study of two NHS hospitals in England. BMJ Qual Saf 2018;27:103-9.

5 Golda N, Beeson S, Kohli N, et al. Analysis of the patient experience measure. J Am Acad Dermatol 2018;78:645-51.

6 Baldie DJ, Guthrie B, Entwistle V, et al. Exploring the impact and use of patients' feedback about their care experiences in general practice settings-a realist synthesis. Fam Pract 2018;35:13-21.

7 Donabedian A. The Lichfield lecture. quality assurance in health care: consumers' role. Qual Health Care 1992;1:247-51.

8 Saut AM, Berssaneti FT. Patient involvement in quality management of healthcare services. Acta Paul Enferm 2016;29:85.

9 Hernan AL, Kloot K, Giles SJ, et al. Investigating the feasibility of a patient feedback tool to improve safety in Australian primary care: a study protocol. BMJ Open 2019;9:e027327-7.

10 Snyder H, Engström J. The antecedents, forms and consequences of patient involvement: a narrative review of the literature. Int J Nurs Stud 2016;53:351-78.

11 Flott KM, Graham C, Darzi A, et al. Can we use patient-reported feedback to drive change? the challenges of using patient-reported feedback and how they might be addressed. BMJ Qual Saf 2017;26:502-7.

12 Berwick DM. Era 3 for medicine and health care. JAMA 2016;315:1329-30.

13 Yin RK. Case study research: design and methods. 5th edition. Thousand Oaks, CA: Sage Publications, 2014: 290.

14 Berssaneti FT, Saut AM, Barakat MF, et al. Is there any link between accreditation programs and the models of organizational excellence? Rev Esc Enferm USP 2016;50:650-7.

15 Reichheld FF. The one number you need to grow. Harv Bus Rev 2003;81:45-54.

16 Taylor N, Clay-Williams R, Hogden E, et al. Deepening our understanding of quality in Australia (DUQuA): a study protocol for a nationwide, multilevel analysis of relationships between hospital quality management systems and patient factors. BMJ Open 2015;5:e010349.

17 Sheard L, Marsh C, O'Hara J, et al. The patient feedback response framework - understanding why UK hospital staff find it difficult to make improvements based on patient feedback: a qualitative study. Soc Sci Med 2017;178:19-27.

18 Rozenblum R, Lisby M, Hockey PM, et al. The patient satisfaction chasm: the gap between hospital management and frontline clinicians. BMJ Qual Saf 2013;22:242-50.

19 Wiig S, Storm M, Aase K, et al. Investigating the use of patient involvement and patient experience in quality improvement in Norway: rhetoric or reality? BMC Health Serv Res 2013;13:14

20 Secanell M, Groene O, Arah OA, et al. Deepening our understanding of quality improvement in Europe (DUQuE): overview of a study of hospital quality management in seven countries. Int J Qual Health Care 2014;26 Suppl 1:5-15.

21 Parand A, Dopson S, Renz A, et al. The role of hospital managers in quality and patient safety: a systematic review. BMJ Open 2014;4:e005055.
22 Boiko O, Campbell JL, Elmore N, et al. The role of patient experience surveys in quality assurance and improvement: a focus group study in English general practice. Health Expect 2015;18:1982-94.

23 Gleeson $\mathrm{H}$, Calderon A, Swami V, et al. Systematic review of approaches to using patient experience data for quality improvement in healthcare settings. BMJ Open 2016;6:e011907.

24 Baines R, Regan de Bere S, Stevens S, et al. The impact of patient feedback on the medical performance of qualified doctors: a systematic review. BMC Med Educ 2018;18:173.

25 Parry GJ. A brief history of quality improvement. special series: quality care symposium. J Oncol Pract 2014;10:196-9.

26 Nordin N, Radzi WNWM, Razak RC, et al. Complaints and compliments assessment in developing service delivery measurement. Manag Sci Lett 2018;8:293-304.

27 Boyce MB, Browne JP, Greenhalgh J. The experiences of professionals with using information from patient-reported outcome measures to improve the quality of healthcare: a systematic review of qualitative research. BMJ Qual Saf 2014;23:508-18.

28 Bodenheimer T, Sinsky C. From triple to quadruple aim: care of the patient requires care of the provider. Ann Fam Med 2014;12:573-6.

29 Coulter A, Locock L, Ziebland S, et al. Collecting data on patient experience is not enough: they must be used to improve care. BMJ 2014;348:g2225-4.

30 Coulter A. Measuring what matters to patients. BMJ 2017;356:j816-2.

31 Farley $\mathrm{H}$, Enguidanos ER, Coletti CM, et al. Patient satisfaction surveys and quality of care: an information paper. Ann Emerg Med 2014;64:351-7.

32 Mittinty MM, Lee J, de C Williams AC, et al. Exploring patient experiences of a pain management centre: a qualitative study. Scand J Pain 2017:17:378-81.

33 Reader TW, Gillespie A, Roberts J. Patient complaints in healthcare systems: a systematic review and coding taxonomy. BMJ Qual Saf 2014;23:678-89.

34 de Vos MS, Hamming JF, Marang-van de Mheen PJ. The problem with using patient complaints for improvement. BMJ Qual Saf 2018;27:758-62

35 Gill SD, Redden-Hoare J, Dunning TL, et al. Health services should collect feedback from inpatients at the point of service: opinions from patients and staff in acute and subacute facilities. Int J Qual Health Care 2015;27:507-12.

36 Hawkins CM, DeLaO AJ, Hung C. Social media and the patient experience. J Am Coll Radiol 2016;13:1615-21.

37 Lagu T, Goff SL, Craft B, et al. Can social media be used as a hospital quality improvement tool? J Hosp Med 2016;11:52-5.

38 Rozenblum R, Bates DW. Patient-Centred healthcare, social media and the Internet: the perfect storm? BMJ Qual Saf 2013;22:183-6.

39 Lafortune C, Elliott J, Egan MY, et al. The rest of the story: a qualitative study of complementing standardized assessment data with informal interviews with older patients and families. Patient 2017;10:215-24.

40 Beattie M, Murphy DJ, Atherton I, et al. Instruments to measure patient experience of healthcare quality in hospitals: a systematic review. Syst Rev 2015;4:97.

41 Manary MP, Boulding W, Staelin R, et al. The patient experience and health outcomes. N Engl J Med 2013;368:201-3.

42 Saunders CL, Elliott MN, Lyratzopoulos G, et al. Do differential response rates to patient surveys between organizations lead to unfair performance comparisons?: evidence from the English cancer patient experience survey. Med Care 2016;54:45-54-54.

43 Anhang Price R, Elliott MN, Zaslavsky AM, et al. Examining the role of patient experience surveys in measuring health care quality. Med Care Res Rev 2014;71:522-54.

44 Golda N, Beeson S, Kohli N, et al. Recommendations for improving the patient experience in specialty encounters. J Am Acad Dermatol 2018;78:653-9.

45 Reason J. Understanding adverse events: human factors. Qual Health Care 1995;4:80-9-9.

46 Reason J. Human error: models and management. BMJ 2000;320:768-70.

47 Kahneman D, Tversky A. Subjective probability: a judgment of representativeness. Cogn Psychol 1972;3:430-54.

48 Lima TdeM, Aguiar PM, Storpirtis S. Development and validation of key performance indicators for medication management services provided for outpatients. Res Soc Adm Pharm 2019;15:1080-7.

49 Perla RJ, Provost LP, Parry GJ. Seven propositions of the science of improvement: exploring foundations. Qual Manag Health Care 2013;22:170-86-86. 